\title{
Compresión de Señales Electrocardiográficas Fetales Mediante la Transformada Wavelet Packet
}

\author{
Gonzalo Jimenez ${ }^{(1)}$. Edwin Rivas ${ }^{(2)}$ y Lilia E. Aparício(1) \\ Universidad Distrital FJC, Facultad de Ingeniería, (1) Dpto. de Ing. Electrónica, (2) Dpto. de Ing. Eléctrica, \\ Bogota -Colombia (e-mail:gjimenezp@udistrital.edu.co;gojimenez1@hotmail.com)
}

Recibido Sep. 1, 2017; Aceptado Nov. 9, 2017; Versión final Ene. 9, 2018, Publicado Jun. 2018

\begin{abstract}
Resumen
Este artículo presenta el desarrollo y evaluación de un algoritmo para la compresión de señales electrocardiográficas fetales, tomadas de manera superficial sobre el abdomen materno. Este proceso produce un gran volumen de información que dificulta su almacenamiento y/o trasmisión. El algoritmo propuesto busca una compresión sin pérdidas de la señal, aplicando Transformada Wavelet Packet con el fin de mantener un error por debajo de la unidad, con relaciones de compresión superiores 20:1 y con la energía conservada en la reconstrucción como parámetro de comparación. Para la validación del algoritmo se hace uso de los archivos de señales proporcionados en la base de datos PhysioBank DataBase.
\end{abstract}

Palabras clave: conservación de energía; CR; ECG; Fetal; PDR; transformada wavelet packet

\section{Compression of fetal electrocardiographic signals using the Wavelet Packet transform}

\begin{abstract}
This article presents the development and evaluation of an algorithm for the compression of fetal electrocardiographic signals, taken superficially on the maternal abdomen. This process produces a large volume of information that makes it difficult to store and / or transmit. The proposed algorithm seeks a lossless compression of the signal, applying Wavelet Packet Transform in order to maintain an error below the unit, with compression ratios higher than 20: 1 and with the energy conserved in the reconstruction as a comparison parameter. For the validation of the algorithm, use is made of the signal files provided in the PhysioBank DataBase.
\end{abstract}

Keywords: conserved energy; CR; ECG Fetal; wavelet packet transform 


\section{INTRODUCCIÓN}

La caracterización de señales electrocardiográficas fetales pretenden diagnosticar posibles enfermedades que coloquen en riesgo la vida del feto, como se explica en Rajesh y Ganesan (2014) en la literatura científica se reportan los métodos invasivo y no invasivo. El método invasivo consiste en colocar un electrodo en el cuero cabelludo del feto, mientras que el método no invasivo mostrado por ZhiLin (2013) consiste en colocar electrodos sobre la superficie abdominal de la madre para capturar la señal ECG (electrocardiograma) fetal, esto exige la detección y separación de las señales, fetal y materna, lo cual representa un problema de difícil solución debido a que estas señales están rodeadas de factores como el ruido producido por el movimiento muscular, la interferencia de la señal materna, el ruido eléctrico producido por los instrumentos de medida y el movimiento fetal; en Panigrahy, Rakshit (2015) se expone que estos factores cambian las características de potencia de la señal que de por si es muy baja.

La presencia de ruido en esta señal y la interferencia de la señal materna hacen que se produzca un volumen considerable de datos, y como se muestra en Arvinti y Costache (2014), esto es un factor a tener en cuenta en la caracterización o análisis de la señal; con una frecuencia de muestreo de $360 \mathrm{~Hz}$, un registro de 24 horas requiere 43 Mbytes. A fin de lograr el nivel de compresión deseado, de acuerdo a las necesidades y requerimientos del medio de almacenamiento o el ancho de banda de trasmisión, se fija un umbral para el tipo y la cantidad de datos. Como se expone en Brechet (2007) existen básicamente dos métodos de compresión. Estos son sin perdidas y con pérdidas. Como se muestra en la mayoría de literatura científica aplican una compresión con pérdidas con una relación de compresión que está por encima de 20:1. Pero un error en la reconstrucción entre el 50 y $60 \%$.

Como se muestra en Castillo, Morales (2013), uno de los problemas es la selección de un umbral adecuado, para eliminar los niveles de ruido en las señales ECG dado su no linealidad y más particularmente en las señales ECG fetales. En este trabajo como método de selección del umbral se aplica la cantidad de energía conservada en la reconstrucción de la señal, método expuesto en Rivas y Burgos (2009), lo que permite mejorar los valores de compresión, manteniendo un error por debajo de la unidad porcentual.

\section{MARCO CONCEPTUAL}

El tratamiento de una señal electrocardiográfica fetal implica la obtención, adecuación, captura del complejo QRS y procesamiento de la señal. En la figura 1 se presenta un diagrama de bloques del tratamiento generalmente presentado en la literatura científica según se presenta en Chen y Cheng (2015), de una señal ECG. Desde hace varios años se ha venido dando uso a la transformada Wavelet en diferentes campos como la compresión de información y la eliminación de ruido como en Santamaría (2012). Las señales se obtienen de Goldemberg, Amaral et al (2000). Estas señales constan de registros que tienen una duración hasta de 4 horas muestreados a $1 \mathrm{KHz}$ por dos canales. Estos registros además de las señales contienen información médica relacionada con las señales.

En primera instancia se adecua la señal a través del filtrado, a fin de eliminar ruido eléctrico y la separación de la señal ECG materna. Como se ve en Rao y Yao (2015) las principales técnicas de extracción de las señales son filtros adaptativos, descomposición lineal y no lineal; posteriormente se captura el complejo QRS, que es el objeto de estudio y finalmente se procesa la señal utilizando para ello método estadísticos de las características de la señal o por la transformación de la señal como por ejemplo en Lima y Alvarado (2016) o en Ebrajimzadeh (2011) donde se aplica Wavelets. Otra técnica expuesta en Gerla (2009) es la separación siega de fuentes, para la extracción de las características de la misma.

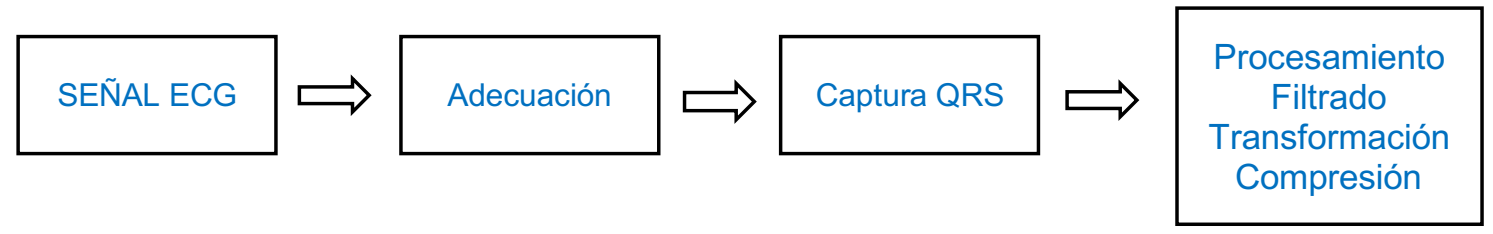

Fig. 1: Diagrama de bloques de tratamiento de señales ECG

La selección de la transformada juega un papel importante en el análisis y procesamiento de las señales, como se demuestra en Craven (2016). Para llevar a cabo esta labor normalmente se realiza la comparación visual de la señal a tratar con una serie de transformadas o se realiza un análisis de correlación entre la señal y la transformada. Existen tres requisitos que debe llenar toda transformada Wavelet, ellos son: un valor promedio nulo, su energía debe ser finita y ortogonalidad para que sea admisible. Matemáticamente se expresan estas condiciones en las ecuaciones 1,2 y 3. 


$$
\begin{aligned}
& \int_{-\infty}^{\infty} \Psi(t) d t=0 \\
& \int_{-\infty}^{\infty}|\Psi(t)|^{2} d t<\infty \\
& C_{\Psi}=\frac{1}{\sqrt{2 \pi}} \int_{-\infty}^{\infty} \frac{|\widehat{\Psi}(w)|^{2}}{|w|} d w<\infty
\end{aligned}
$$

En esta investigación se propone la comparación teniendo en cuenta la cantidad de energía conservada después de la reconstrucción. Esta energía se ve afectada por los niveles de descomposición de las señales ECG fetales.

Las principales características de una transformada son:

Los momentos de desvanecimiento de una Wavelet está relacionado con el orden de la función, así una función tiene $\mathrm{N}$ momentos de desvanecimiento si tiene un orden $\mathrm{N}$. matemáticamente se expresa como lo muestra la ecuación 4.

$$
\int_{-\infty}^{\infty} \Psi^{\mathrm{i}} \mathrm{dt}=0
$$

Si se cumple (4) entonces para $\mathrm{i}=0,1,2, \ldots . \mathrm{N}-1$, donde i es el iesimo momento de desvanecimiento, así existen $\mathrm{N}-1$ momentos de desvanecimiento para un orden $\mathrm{N}$. También la cantidad de momentos de desvanecimiento muestra o está relacionado con la selectividad de la descomposición Wavelet. El tamaño del soporte se relaciona con el orden de la transformada y por lo tanto con los momentos de desvanecimiento así: el tamaño del soporte es $2 \mathrm{~N}-1$. La regularidad lo que representa es la suavidad de la transformada wavelet, esto sirve para la reconstrucción de la señal estudiada. La función de escala es una función ortonormal a la función wavelet. Si no existe una función de escala no es posible utilizar esta familia wavelet para discretizar la señal.

Este trabajo utiliza como base de selección de la transformada, la cantidad de energía conservada después de la reconstrucción de la señal, como se observa en la figura 2, se mantiene un nivel de energía del 99,99\%

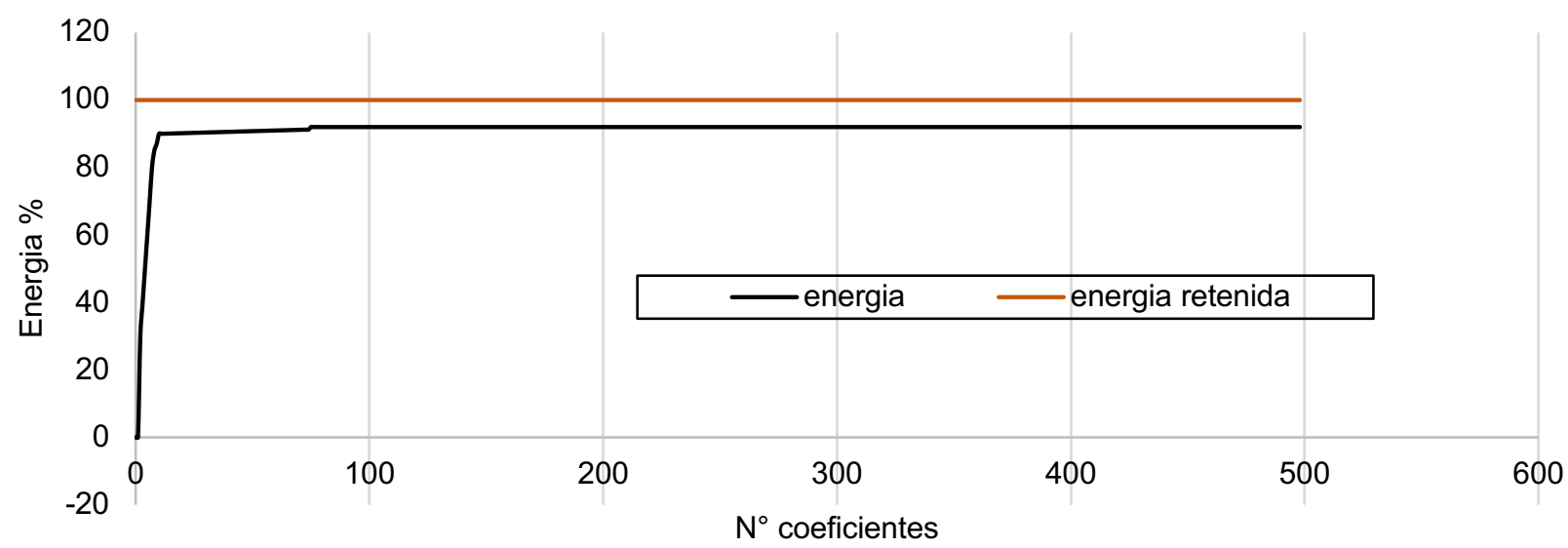

Fig. 2: Grafica del número de coeficientes utilizados con respecto de la energía conservada en la reconstrucción.

Las señales electrocardiográficas fetales que se utilizaron fueron tomadas de la base de datos MITH DATA BASE del PhysioBank ATM de physionet provista por Goldemberg, Amaral et al (2000). Para la compresión de estas se seleccionó la wavelet Daubechies, como se ve en Singh (2015), muestran un mejor rendimiento en el análisis de señales ECG. 


\section{Métodos de compresión}

El estudio comparativo de los métodos de compresión presentado en Chae (2013) muestra que la búsqueda de la simplicidad en el codificador permite menor complejidad en la compresión, medida en el consumo de memoria para el proceso y el tiempo de respuesta, pero que se debe tener cuidado cuando se trata de señales de bajo nivel y embebidas en ambientes ruidosos, producidos por el movimiento del feto, las contracciones uterinas y los producidos por los propios electrodos sobre la piel, como es el caso de las señales ECG fetales en Zhang (2016) se presentan diferentes tipos de umbralización en presencia de ruido no blanco llegando a la conclusión de la umbralización flexible y umbral por cada nivel presenta la menor relación de error. La tabla 1 muestra una comparación de resultados de CR (relación de compresión) y PDR (porcentaje de error en la reconstrucción) para diferentes métodos de compresión, tipo de señal y con las herramientas utilizadas.

Tabla 1: Resultados comparativos de diferentes métodos de compresión con las herramientas utilizadas en el proceso.

\begin{tabular}{|c|c|c|c|c|c|c|c|c|}
\hline Autor & Perdida & fetal & Preprocesamiento & umbralización & Cuantización & Codificación & $\mathrm{CR} \%$ & $\begin{array}{l}\text { PRD } \\
\%\end{array}$ \\
\hline Jarisch (1980) & Con & $\mathrm{Si}$ & Filtro Kalman & No & No & No & No & No \\
\hline $\begin{array}{l}\text { Mukhopadhya } \\
\text { y (2013) }\end{array}$ & Con & No & T Hilbert & No & No & No & 7,18 & 0,023 \\
\hline ZhiLin (2013) & Con & $\mathrm{Si}$ & No & No & No & $\begin{array}{l}\text { Bayesian } \\
\text { learning }\end{array}$ & No & No \\
\hline $\begin{array}{l}\text { Jin Wang } \\
(2010)\end{array}$ & Con & No & Wavelet & No & No & $\begin{array}{l}\text { Neural } \\
\text { network }\end{array}$ & 7,6 & 2,74 \\
\hline $\begin{array}{l}\text { Ebrajimzadeh } \\
(2011)\end{array}$ & Con & No & Wavelet & No & $\begin{array}{l}\text { Tres niveles } \\
\text { de } \\
\text { cuantización }\end{array}$ & Huffman & 13,92 & 0,97 \\
\hline Arvinti (2011) & Con & No & $\begin{array}{c}\text { Banco de filtros } \\
\text { DWT }\end{array}$ & No & No & No & 7,79 & No \\
\hline $\begin{array}{l}\text { Honteng } \\
(2011)\end{array}$ & Con & No & Wave atom & No & $\begin{array}{c}\text { Dos niveles } \\
\text { de } \\
\text { cuantización }\end{array}$ & Huffman & 10,45 & 0,997 \\
\hline $\begin{array}{l}\text { Jayashree } \\
\text { (2011) }\end{array}$ & Con & No & No & No & DWT & PDLZW & 8,68 & 0,14 \\
\hline $\begin{array}{c}\text { Hernando } \\
(2011) \\
\end{array}$ & Con & No & DWT & No & N-PR CMFB & No & 0,58 & No \\
\hline Seong (2012) & Con & No & DWT & No & No & No & 5,5 & 5,33 \\
\hline $\begin{array}{l}\text { Zhicheng Li } \\
(2015)\end{array}$ & Con & No & Wavelet & No & No & $\begin{array}{l}\text { Compressed } \\
\text { Sensing }\end{array}$ & 4 & \\
\hline $\begin{array}{c}\text { Chandan } \\
\text { Kumar Jha } \\
(2016)\end{array}$ & Con & No & $\begin{array}{l}\text { Filtro Savitzky- } \\
\text { Golay }\end{array}$ & No & Wavelet & $\begin{array}{l}\text { Run Length } \\
\text { Encoding }\end{array}$ & 44 & 0,36 \\
\hline $\begin{array}{c}\text { Chandan } \\
\text { Kumar Jha } \\
(2015)\end{array}$ & Con & No & $\begin{array}{c}\text { Filtro pasa banda } \\
\text { y pasa alto }\end{array}$ & No & Wavelet & $x$ & 90,23 & 0,34 \\
\hline $\begin{array}{l}\text { B. Arvinti } \\
(2014)\end{array}$ & $\operatorname{Sin}$ & No & No & No & $\begin{array}{l}\text { Daubichies1 } \\
8\end{array}$ & $\begin{array}{l}\text { Daubichies1 } \\
8\end{array}$ & 14,98 & No \\
\hline $\begin{array}{l}\text { Motinath } \\
(2016)\end{array}$ & $\operatorname{Sin}$ & No & Saviky & No & Wavelet & $\begin{array}{l}\text { WDT(db1- } \\
40)\end{array}$ & 15,2 & 0,23 \\
\hline Wang (2016) & Sin & No & No & No & Wavelet & Db1-16 & 14 & 0,97 \\
\hline $\begin{array}{c}\text { Jiménez, } \\
\text { Rivas método } \\
\text { propuesto }\end{array}$ & $\operatorname{Sin}$ & $\mathrm{SI}$ & Filtro FIR & universal & wavelet & Db1-10 & 25 & 0,26 \\
\hline
\end{tabular}

\section{Selección del umbral}

Otro parámetro crítico en la compresión de señales es la selección del umbral. En la búsqueda de una compresión sin perdidas, en este trabajo se ha seleccionado el umbral duro, ecuación 5.

$$
s(x)=\left\{\begin{array}{ll}
\mathrm{s}(\mathrm{x}), & |\mathrm{x}|>\delta \\
0 & ,|\mathrm{x}| \leq \delta
\end{array}\right\}
$$

Donde $\mathrm{s}(\mathrm{x})$ es la función a analizar y y $\delta$ es el umbral propuesto. 


\section{Parámetros de comparación}

En la ecuación 6 se muestra un parámetro de medida que tiene en cuenta la relación de compresión (CR). Consiste básicamente en la comparación entre el Tamaño de la señal original y la señal comprimida.

$$
C R=\frac{L_{\text {señal comprimida }}}{L_{\text {señal original }}}
$$

Y la similitud entre la señal original y la señal reconstruida, dado por (Percent Root-Mean-Square Distortion) PDR, mostrada en la ecuación 7.

$$
\operatorname{PDR}(\%)=\sqrt{\frac{\sum_{\mathrm{n}=1}^{\mathrm{N}}\left(\mathrm{S}_{\mathrm{n}}-\widetilde{\mathrm{S}_{\mathrm{n}}}\right)^{2}}{\sum_{\mathrm{n}=1}^{\mathrm{N}} \mathrm{S}_{\mathrm{n}}{ }^{2}}}
$$

Donde S y $\tilde{S}$ son respectivamente la señal original y la señal reconstruida.

$$
Q S=\frac{C R}{P D R}
$$

La calidad de la señal QS, ecuación 8, se obtiene de la relación entre la relación de compresión y el porcentaje de error de la reconstrucción al comparar la señal original y la señal reconstruida.

\section{METODOLOGIA PROPUESTA}

La metodología propuesta consiste en la selección de una transformada dentro de la familia de las Daubechies, que permita que la relación de compresión CR alcance los valores más altos, en este caso un valor superior a 20:1. Para lograr este resultado se fija un valor de energía retenida en la reconstrucción del $99,99 \%$.

En la figura 3 se presenta un esquema de los pasos del algoritmo propuesto. El primer paso de es el filtrado de la señal, para eliminación de ruido eléctrico y establecimiento de línea de base. Este filtro es un filtro tipo FIR de orden 3. Para la selección de la wavelet madre se fija el nivel de energía que garantiza un PDR de nivel por debajo del $1 \%$. El umbral permite seleccionar los coeficientes que se tienen en cuenta para la compresión. En este caso el umbral utilizado es el umbral universal. Se realiza la compresión de la señal y luego se calcula la relación de compresión. El siguiente paso consiste en la reconstrucción de la señal y el cálculo de PDR. Se compara los valores de los parámetros medidos y si es el caso se ajusta el nivel de energía para lograr un valor de CR que este cercano por encima a 20:1, que es lo que en este trabajo se busca. Como se muestra en Singh (2015) y se corrobora en la tabla 3 la familia de transformadas que mejores rendimientos de compresión de señales ECG da es la Daubechies. Se obtienen valores para CR bajos 10:1 cuando se Db2 y el valor máximo obtenido de 25:1 para Db6. Y dos niveles de descomposición.

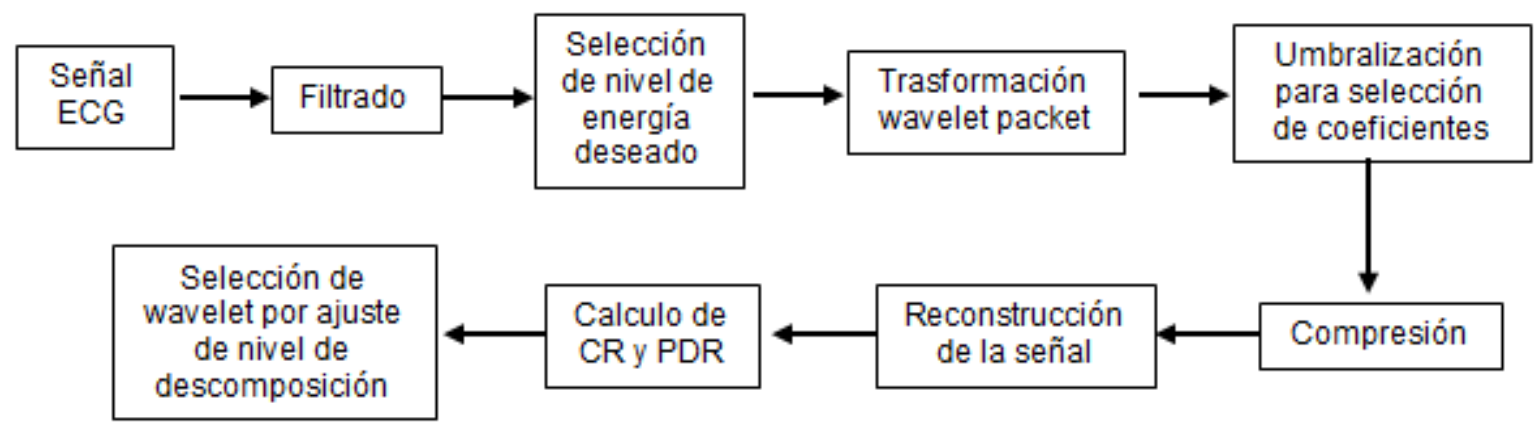

Fig. 3: Diagrama de bloques de algoritmo propuesto

Para la selección de la wavelet madre se inicia con Daubechies de orden 1. Este orden puede variar de 1 a 45, pero experimentalmente se comprobó que para valores de orden superior a 6 , la relación de compresión no aumenta significativamente pero el PDR si empieza a subir. 
Tabla 2: Propiedades de la wavelet madre Daubechies

\begin{tabular}{|l|l|}
\hline Propiedad & Daubechies \\
\hline Orden & $1,2, \ldots 45^{2}$ \\
\hline Regularidad & Relativa \\
\hline Tamaño de soporte & $2 \mathrm{~N}-1$ \\
\hline Longitud del filtro & $2 \mathrm{~N}$ \\
\hline Simetría & $\mathrm{Si}$ \\
\hline Momentos de desvanecimiento & $\mathrm{N}$ \\
\hline Función de escala & $\mathrm{Si}$ \\
\hline Wavelet continua & Posible \\
\hline Wavelet discreta & Posible \\
\hline Expresión explicita & No \\
\hline
\end{tabular}

\section{RESULTADOS}

Como se observa en la figura 4 en la medida que se aumenta el nivel de descomposición de la señal el CR aumenta, pero después del orden 6 se estabiliza el valor, con la disminución del PDR en comparación con lo observado en la figura dos se logran mejoras en los dos parámetros de rendimiento. En el caso de la CR se alcanzó hasta 25:1 y en PDR se bajó hasta 0,22\%.

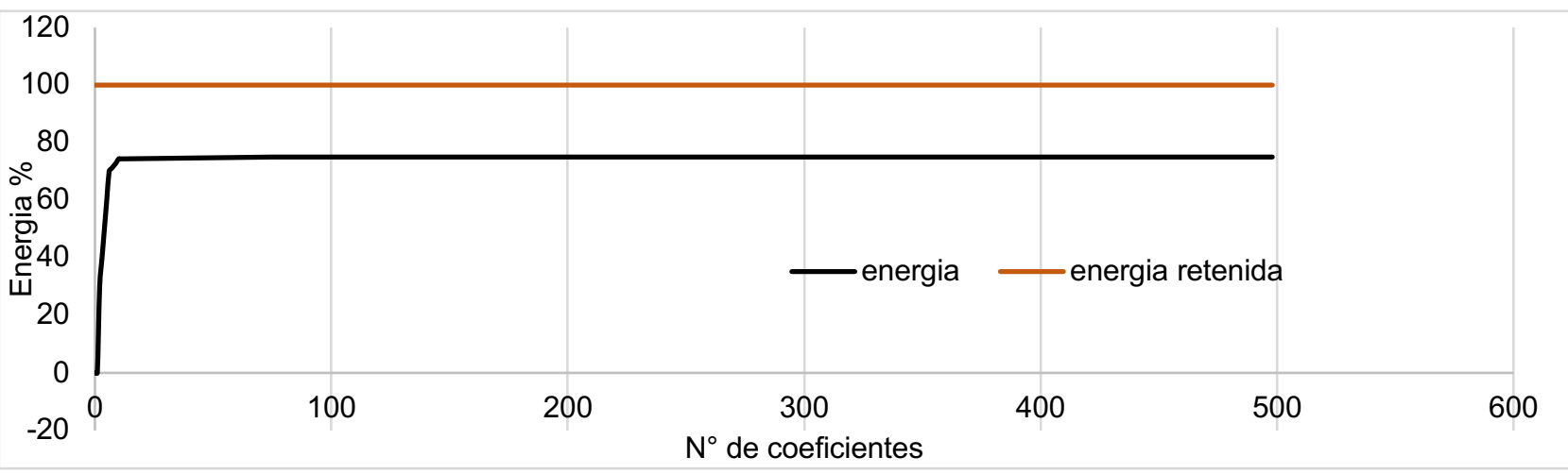

Fig. 4: Muestra de la comparación de valor de compresión CR, complemento del valor mostrado de mostrado de ceros con respecto al PDR identificado como la energía retenida.

La figura 5 muestra la comparación entre la relación de compresión y la PDR para los valores obtenidos en diferentes registros. También se puede observar en la misma figura como los valores de relación de compresión se mantienen cercanos a 20 en su mayoría mientras que los valores de error en la reconstrucción están por debajo de la unidad porcentual; para algunos valores no se cumple debido a que son los más ruidosos. Esto debido a que como se explicó en la metodología para la selección de la wavelet se requiere mantener la mayor cantidad de energía posible después de la reconstrucción. Para evaluar esto se aplicaron a la señal wavelets de diferentes órdenes, desde 1 hasta 10. Debido a esto se toma como este orden como el mejor. En la figura 5 y en la tabla 3 se puede observar que el método propuesto ofrece mejores rendimientos tanto en CR como en PDR

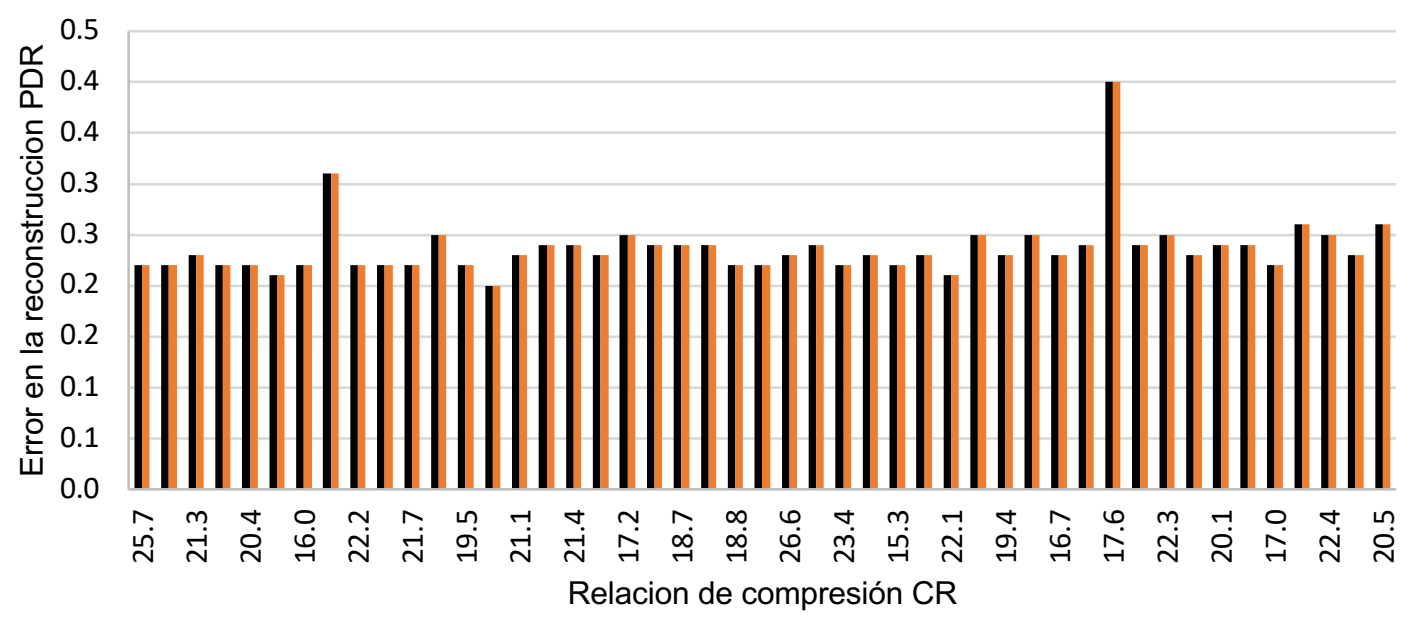

Fig.5: Grafica de comparación (CR) contra (PDR) 
Los valores de relación de compresión de la tabla 3 muestran los resultados de parámetros de medición de rendimiento en compresión, obtenidos por diferentes autores y el método propuesto. Se realiza esta comparación sobre los resultados e técnicas aplicadas al mismo registro. En el caso de otros registros, la relación de compresión CR se vio afectada por el nivel de ruido. Pero en general se logra comprimir la señal en los valores que se habían propuesto al inicio de la investigación y en niveles superiores en comparación con trabajos de otros autores. También se incluye un parámetro que no es comúnmente usado como es el de factor de calidad QS.

Tabla 3: Resultados comparativos de diferentes métodos de compresión.

\begin{tabular}{|l|l|l|l|l|}
\hline AUTOR & REGISTRO & CR\% & PDR\% & QS \\
\hline Lee (2011) & 100 & 9,6 & 0,44 & 21,8181818 \\
\hline Lee (2011) & 100 & 23 & 1,94 & 11,8556701 \\
\hline Ma (2015) & 100 & 15 & 0,29 & 51,7241379 \\
\hline Zhao (2016) & 100 & 14,8 & 7,58 & 1,9525066 \\
\hline Wang (2016) & 100 & 18,16 & 7,25 & 2,50482759 \\
\hline $\begin{array}{l}\text { Jiménez y Rivas } \\
\text { Método propuesto }\end{array}$ & 100 & 25 & 0,22 & 113,636364 \\
\hline
\end{tabular}

La figura 6 muestra la comparación entre la señal original en rojo y la señal comprimida en negro. Se puede ver la semejanza de la señal comprimida con la original.

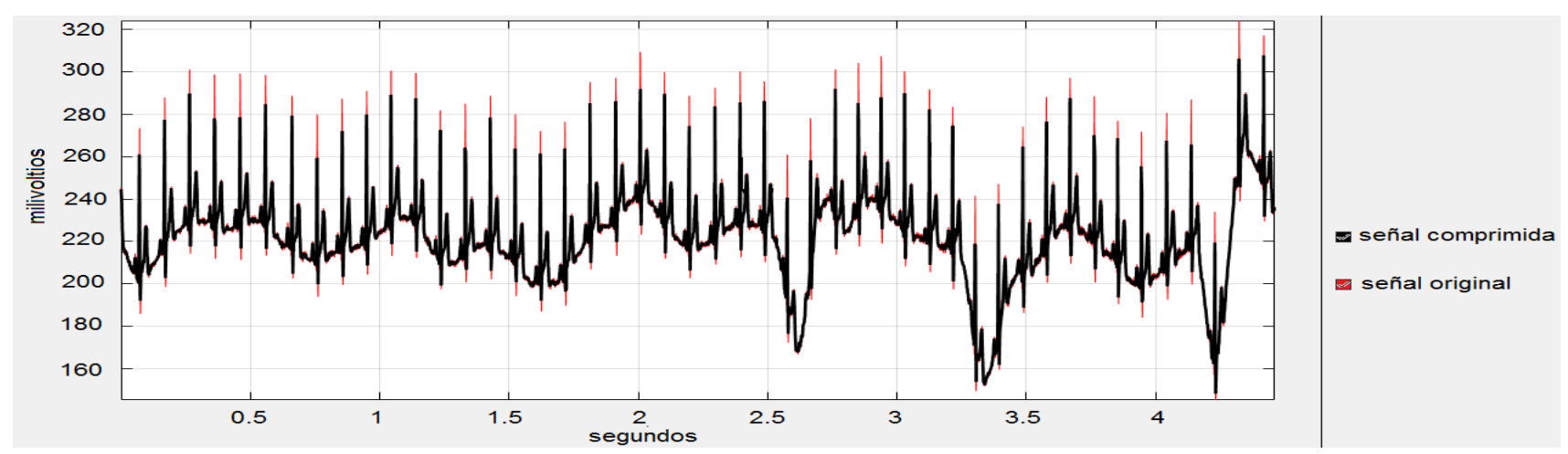

Fig.6: señal original y señal comprimida

En la figura 7 muestra la comparación de resultados de trabajos realizados sobre compresión.

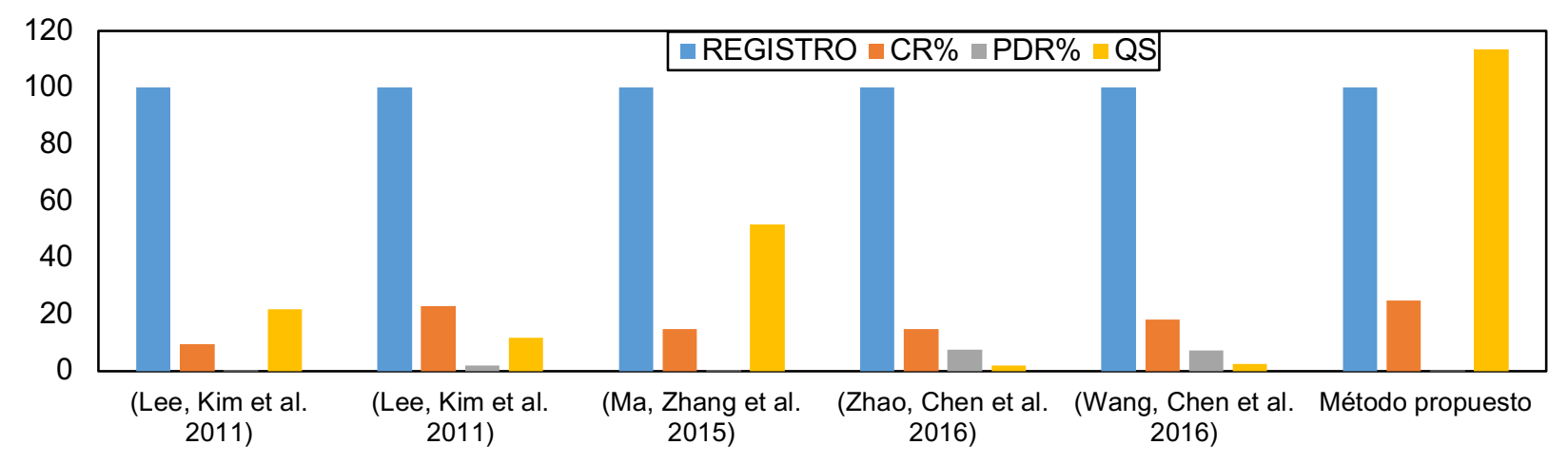

Fig. 7: Grafica de comparación de parámetros CR, PDR y QS

\section{CONCLUSIONES}

Como se observa en Castillo (2013), para mejorar la relación de compresión de la señal el método asociado al uso de la transformada Wavelet, mayormente usado ha estado enfocado con la optimización de la selección del árbol de descomposición. En este trabajo el enfoque está en la selección de la mejor transformada madre. Como se puede ver la selección del umbral de cantidad de energía conservada en la reconstrucción presenta un método diferente al análisis de la relación señal a ruido que comúnmente se usa para la selección de la Wavelet madre aplicada en la compresión. Como se observa en la figura 6 se obtiene mejoras en los niveles de parámetros de comparación seleccionados para el mismo registro. La variación de los parámetros CR Y PDR dependen de la calidad de señal que se requiera y del ancho de banda o medio de almacenamiento del 
que se disponga. El método propuesto tiene un nivel de calidad (QS) mayor, esto debido a que el nivel del PDR es menor al $1 \%$ pero combinado con una rata de compresión mayor a 20 , alcanzando en promedio un valor de 113,63 , lo que se ve en la tabla 3 en la columna QS. Para almacenamiento es importante que la relación de compresión sea alta mientras que para el análisis de frecuencia de la señal se puede bajar la relación de compresión y mantener PDR bajo. El siguiente paso será clasificar mediante una red neuronal los coeficientes que se ajusten a un tipo de patología, observando su relación con la forma de la señal, a fin de poder aumentar la relación de compresión manteniendo el numero original de datos y la energía en la señal reconstruida lo más cercano al $100 \%$.

Debido a los resultados la familia de transformadas que mejor se desempeña en la compresión de las señales ECG fetales es la Daubichies y los niveles de descomposición están entre 2 y 4 niveles para mantener los valores de CR y PDR dentro de los rangos propuestos de 20:1 y $1 \%$ respectivamente.

\section{REFERENCIAS}

Arvinti, B. y M. Costache, The performance of the Daubechies mother wavelets on ECG compression, Electronics and Telecommunications (ISETC) 11th International Symposium on, 1-4, Timisoara, Romania, 14 Noviembre (2014).

Arvinti, B., y A. Isar, An adaptive compression algorithm for ECG signals, Computational Intelligence and Informatics (CINTI), IEEE 12th International Symposium on, 91-95, Budapest, Hungria, 21 Noviembre (2011)

Brechet, L. "Compression of Biomedical Signals With Mother Wavelet Optimization and Best-Basis Wavelet Packet Selection." IEEE Transactions on Biomedical Engineering, 54(12), 2186-2192 (2007).

Castillo, E., D. P. Morales, "Efficient wavelet-based ECG processing for single-lead FHR extraction." Digital Signal Processing 23(6), 1897-1909, (2013).

Craven, D. y B. McGinley, "Energy-efficient Compressed Sensing for ambulatory ECG monitoring." doi: 10.1016/j.compbiomed.2016.01.013. Elsevier, Vol. 71, 1-13 (2016).

Chae, D. H., Y. F. Alem, Performance study of compressive sampling for ECG signal compression in noisy and varying sparsity acquisition. DOI: 10.1109/ICASSP.2013.6637862, IEEE, 1306-1309 (2013).

Chen, Y. y C. Cheng, Reconstruction of sparse multiband wavelet signals from Fourier measurements, International Conference on Sampling Theory and Applications (SampTA), 78-81, Washington, DC, USA 25 Mayo (2015).

Ebrahimzadeh, A. y M. Azarbad, ECG compression using wavelet transform and three-level quantization. Digital Content, Multimedia Technology and its Applications (IDC), 6th International Conference on, 256-254, Seoul, South Korea, 16 Agosto (2010).

Gerla, V. y K. Paul, "Multivariate Analysis of Full-Term Neonatal Polysomnographic Data.", DOI: 10.1109/TITB.2008.2007193, IEEE Transactions on Information Technology in Biomedicine. 13(1), 104-110. (2009).

Goldberger, A. L., L. A. N. Amaral, et al, «PhysioBank, PhysioToolkit, and PhysioNet: Components of a New Research Resource for Complex Physiologic Signals», Vol. 101(23), 215-220, (2000).

Hernando-Ramiro, C. y M. Blanco-Velasco, Efficient thresholding-based ECG compressors for high quality applications using cosine modulated filter banks, Engineering in Medicine and Biology Society EMBC, 7079-7082, 30 de Agosto a 3 de Septiembre (2011).

Hongteng, X. y Z. Guangtao, ECG data compression based on wave atom transform, Multimedia Signal Processing (MMSP), IEEE 13th International Workshop on, 1-5, Boston, MA, USA, 17 a 19 de Octubre (2011).

Jagannath, D. y A. I. Selvakumar, "Superior foetal electrocardiogram signal elicitation using a novel artificial intelligent Bayesian methodology." Applied Soft Computing 37(16) 1002-1017, Amsterdam, Netherlands (2015).

Jarisch, W. y J. S. Detwiler, "Statistical Modeling of Fetal Heart Rate Variability." DOI: 10.1109/TBME.1980.326580, Biomedical Engineering IEEE Transactions BME. 27(10), 582-589, India (1980).

Jha, C. K. y M. H. Kolekar, Efficient ECG data compression and transmission algorithm for telemedicine, DOI: 10.1109/COMSNETS.2016.7439988 8th International Conference on Communication Systems and Networks (COMSNETS), Bangalore, 1-6, India (2016).

Jin, W. y L. Xiaomei, ECG data compression research based on wavelet neural network, Computer, Mechatronics, Control and Electronic Engineering (CMCE), 361-363, Changchun, China 24 Aug (2010).

Lee, S. y J. Kim, "A Real-Time ECG Data Compression and Transmission Algorithm for an e-Health Device." DOI: 10.1109/TBME.2011.2156794, IEEE Transactions on Biomedical Engineering. 58(9), 2448-2455 (2011).

Li, Z. y Y. Deng, ECG signal compressed sensing using the wavelet tree model, 8th International Conference on Biomedical Engineering and Informatics (BMEI), 194-199, Shenyang, China, 14-16 Octubre (2015).

Lima-Herrera, S. L. y C. Alvarado-Serrano, Fetal ECG extraction based on adaptive filters and Wavelet Transform: Validation and application in fetal heart rate variability analysis, 13th International Conference on Electrical Engineering, Computing Science and Automatic Control (CCE),1-6, Ciudad de Mexico, 26-30 de Septiembre (2016). 
Ma, J., T. Zhang, "A Novel ECG Data Compression Method Using Adaptive Fourier Decomposition With Security Guarantee in e-Health Applications", DOI: 10.1109/JBHI.2014.2357841, IEEE Journal of Biomedical and Health Informatics. 19(3), 986-994, (2015).

Mallat, S. G., "A theory for multiresolution signal decomposition: the wavelet representation." IEEE Transactions on Pattern Analysis and Machine Intelligence 11(7), 674-693, (1989).

Motinath, V. A. y C. K. Jha, A novel ECG data compression algorithm using best mother wavelet selection, International Conference on Advances in Computing, Communications and Informatics (ICACCI), 682-686 Jaipur, India 21 a 24 de Septiembre (2016).

Mukhopadhyay, S. K. y M. Mitra, ECG signal processing: Lossless compression, transmission via GSM network and feature extraction using Hilbert transform, Point-of-Care Healthcare Technologies (PHT), 85-88, Bangalore, India (2013).

Panigrahy, D. y M. Rakshit, An efficient method for fetal ECG extraction from single channel abdominal ECG, Industrial Instrumentation and Control (ICIC), International Conference on, IEEE, 1083-1088, Pune, India, 28 a 30 de Mayo (2015).

Rajesh, A. V. y R. Ganesan, Comprehensive study on fetal ECG extraction. Control, Instrumentation, Communication and Computational Technologies (ICCICCT), International Conference on, 1187-1192, Kanyakumari, India, 10 a 11 de Julio (2014).

Rao, Y. y H. Zeng, Estimate MECG from abdominal ECG signals using extended Kalman RTS smoother, Sixth International Conference on Intelligent Control and Information Processing (ICICIP), 73-77, Wuhan, China 26 a 28 de Noviembre (2015).

Rivas, E., y J. C. Burgos, "Condition Assessment of Power OLTC by Vibration Analysis Using Wavelet Transform" DOI: 10.1109/TPWRD.2009.2014268, IEEE Transactions on Power Delivery. 24(2), 687-694, (2009).

Santamaria, F. Cortes, et al, Santamaría, F., C. A. Cortés, et al. (2012). "Uso de la Transformada de Ondeletas (Wavelet Transform) en la Reducción de Ruidos en las Señales de Campo Eléctrico producidas por Rayos.", Informacion tecnologica, vol. 23, 65-78 (2012).

Seong-Beom, C. y L. Young-Dong, Implementation of novel ECG compression algorithm using template matching, Computing and Convergence Technology (ICCCT), 7th International Conference on, 305-308, Seoul, South Korea, 3 a 5 Diciembre (2012).

Singh, O. y R. K. Sunkaria, The utility of wavelet packet transform in QRS complex detection - a comparative study of different mother wavelets, 2nd International Conference on Computing for Sustainable Global Development (INDIACom),1942-1947, 11 a 13 de Marzo (2015).

Surekha, K. S. y B. P. Patil, ECG signal compression using hybrid 1D and 2D wavelet transform, Science and Information Conference (SAl), 468-472, London, UK 27 a 29 de Agosto (2014).

Wang, X. y Z. Chen, "ECG compression based on combining of EMD and wavelet transform." DOI: 10.1049/el.2016.2174, Electronics Letters. 52(19), 1588-1590 (2016).

Yao, Z. y Y. Dong, Experimental evaluations of sequential adaptive processing for fetal electrocardiograms (ECGs), 49th Asilomar Conference on Signals, Systems and Computers,770-774, Pacific Grove, CA, USA 8 a 11 de Noviembre (2015).

Zhang, Y. y B. Liu, "Classification of EEG Signals Based on Autoregressive Model and Wavelet Packet Decomposition", Neural Processing Letters, 45(14), 365-378 (2016).

Zhao, C. y Z. Chen,"Electrocardiograph compression based on sifting process of empirical mode decomposition." Electronics Letters, Issue 9, Volume 52 (3), 688-690 (2016).

ZhiLin, Z. y J. Tzyy-Ping, "Compressed Sensing for Energy-Efficient Wireless Telemonitoring of Noninvasive Fetal ECG Via Block Sparse Bayesian Learning." DOI: 10.1109/TBME.2012.2226175, Biomedical Engineering, IEEE Transactions on. 60(2), 300-309 (2013). 
